\title{
Frequency-dependent multiconductor line model based on the Bergeron method
}

\author{
Pablo Torrez Caballero a , Eduardo C. Marques Costa ${ }^{\mathrm{b}}$, Sérgio Kurokawa ${ }^{\mathrm{a}, *}$ \\ a Universidade Estadual Paulista-UNESP, Faculdade de Engenharia de Ilha Solteira-FEIS, Departamento de Engenharia Elétrica, Ilha Solteira, SP, Brazil \\ ${ }^{\mathrm{b}}$ Universidade de São Paulo-USP, Escola Politécnica, Departamento de Engenharia de Energia e Automação Elétricas-PEA, São Paulo, SP, Brazil
}

\section{A R T I C L E I N F O}

\section{Article history:}

Received 5 March 2015

Received in revised form 16 May 2015

Accepted 23 May 2015

Available online 27 June 2015

\section{Keywords:}

Electromagnetic transients

Transmission line modeling

Vector fitting

Time-domain analysis

\begin{abstract}
A B S T R A C T
A new multiconductor transmission line model is proposed based on the method of characteristics. The conventional Bergeron model is characterized by the line representation using constant lumped parameters. The novel content of this paper is the inclusion of the frequency effect in the longitudinal parameters by fitting techniques and three-phase representation of the Bergeron line model. This new feature enables to extend the application of the Bergeron method for simulation of electromagnetic transient in three-phase systems, considering fast and impulsive transients composed of a wide range of frequencies.
\end{abstract}

(c) 2015 Published by Elsevier B.V.

\section{Introduction}

The method of the characteristics, or Bergeron method, was initially applied to solve hydraulic systems and after for electrical problems in electromagnetic propagation through a lossless wave guide [1]. At the same time, end of the 1960s and beginning of the 1970s, two important researches on transmission line modeling-TLM were published. The first work was published by $A$. Budner, which described a two-phase transmission line modeled by a two-port circuit in the frequency-domain. The timedomain currents and voltages at the sending and receiving ends of the two-phase line were calculated using inverse transforms and convolutions [2]. The second important research described the inclusion of the line losses in the Bergeron model using lumped resistances in the equivalent line circuit [3]. The main difference of the two methods is that the Budner's model is based on the representation of the distributed characteristics of the line parameters in the frequency domain whereas the Bergeron line model is a time-domain representation based on lumped circuits without inverse transforms and convolutions. Surely that at the same time there were other important contemporary studies, such as the papers published by H. W. Dommel on the Electromagnetic Transient Program (EMTP) [4]. However, the two prior references are

\footnotetext{
* Corresponding author. Tel.:+55 1130915152.

E-mail addresses: pablotorrezcaballero@gmail.com (P.T. Caballero), educosta@pea.usp.com (E.C.M. Costa),kurokawa@dee.feis.unesp.br (S. Kurokawa).
}

more important specifically for the line model proposed in this research.

An effective evolution in TLM was verified in the 1980s with the great improvement of the computational resources and processing power $[5,6]$. The well-established line model of J. Marti, available in various programs derived from the EMTP, was published in 1982 [7]. This paper introduced in the technical literature on TLM the concept of synthesis of the line parameters, i.e., the frequencydependent characteristic impedance of the line was represented in the time domain as an equivalent circuit composed of resistances and capacitances. Thus, the inverse transforms and convolution used in the first frequency-dependent line models were not more necessary for time-domain transient simulations [2]. At the same period, the Bergeron line model with losses was integrated to tool boxes of power systems available in several simulation programs derived from the EMTP [6].

In the 1990s, important researches were published improving the multiconductor representation of transmission lines and new methods for inclusion of the frequency effect in the line parameters, directly in the time domain, were also included in the TLM literature. The multiphase representation of transmission lines was carried out using modal decoupling, i.e., each phase of a transmission line was decoupled as an independent propagation mode [8]. Thus, there are no mutual parameters in the modal domain and each propagation mode can be considered as a single-phase transmission line. At the same period, fitting techniques were proposed to include the frequency effect on the line longitudinal parameters directly in the time domain. The premise of the technique 


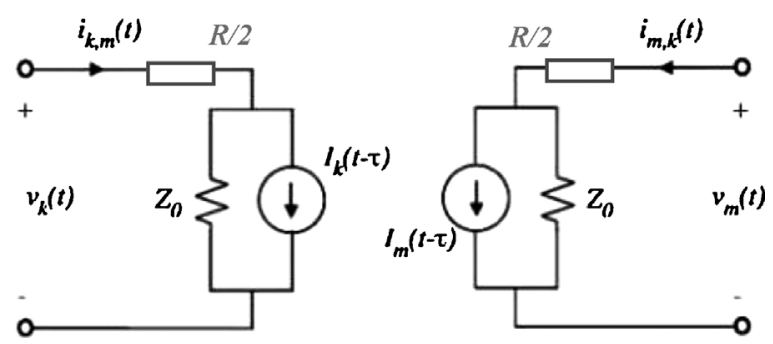

Fig. 1. Bergeron circuit considering the line losses.

denominated vector fitting is that the frequency-dependent resistance and the inductance parameters of transmission lines can be approximated by a rational function and after represented by an equivalent electric circuit in the time domain, such as in the J. Marti line model $[9,10]$. From these two techniques, modal decoupling and vector fitting, several line models were developed [11-15].

In reference [14], modal decoupling and line parameters fitting were applied for development of a three-phase transmission line model in the time domain. Initially, the three-phase line was decoupled into three independent propagation modes that were modeled as three single-phase lines using a frequency-dependent cascade of $\pi$ circuits based on the modal parameters fitting. The time-domain differential equations of currents and voltages along the cascade of $\pi$ circuits were represented as state equations and solved using integration methods.

Recently, the same fitting procedure was applied to the well-known Bergeron line model [15]. Although the frequencydependent approach of the Bergeron model showed accurate results for electromagnetic transient simulations, it was developed for single-phase transmission lines. In the current research, a multiphase representation is developed for the frequency-dependent Bergeron model using an accurate modal decoupling and timedomain modeling by fitting techniques. In sequence, the differential equations of each propagation mode can be represented as state equations and solved using any integration method.

The Bergeron line model is one of the most simple and practical line models available in the EMTP. The frequency-dependent model proposed in this research is free of high-frequency oscillations, as verified in previous line models based on fitting techniques and lumped parameters $[7,10,11,14]$. Furthermore, the proposed Bergeron model is developed based on a unique equivalent circuit, differently of most of the time-domain models that are developed considering a cascade of frequency-dependent circuits in series $[11,14]$. Another contribution with the state of the art is that timevariable events and other non-linear power components can be easily integrated in the proposed line model and during the simulation process [7].

\section{Inclusion of the frequency effect in the Bergeron line model}

The Bergeron line model with losses can be represented concentrating the losses at the two terminals of the line representation. Lumped resistances are included at the sending and receiving ends of the Bergeron circuit, as in Fig. 1.

Currents in Fig. 1 are expressed in (1) and (2) [3]:

$$
\begin{aligned}
& I_{k}(t-\tau)=-\frac{1}{\left(Z_{0}+R / 2\right)} v_{m}(t-\tau)-i_{m, k}(t-\tau) \\
& I_{m}(t-\tau)=-\frac{1}{\left(Z_{0}+R / 2\right)} v_{k}(t-\tau)-i_{k, m}(t-\tau)
\end{aligned}
$$

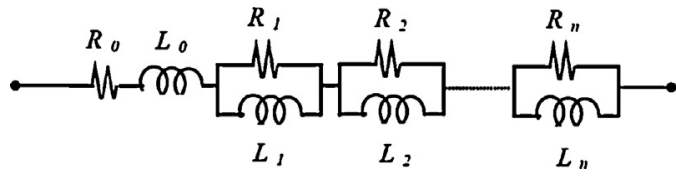

Fig. 2. Time-domain fitting of the line longitudinal parameters.

The characteristic impedance $Z_{0}$ and the propagation time $\tau$, from the sending end to the receiving end of the line, are constant and expressed as:

$Z_{0}=\sqrt{\frac{L^{\prime}}{C^{\prime}}} ; \quad \tau=\frac{l}{v}=l \sqrt{L^{\prime} C^{\prime}}$

Terms $l$ and $v$ are the line length and propagation speed, respectively. The last term is constant and expressed as follows:

$v=\frac{1}{\sqrt{L^{\prime} C^{\prime}}}$

In (1) and (2), term $R / 2$ is added to the characteristic impedance $Z_{0}$ that is a function of the line inductance and capacitance per unit of length (p.u.l.), $L$ ' and $C^{\prime}$, respectively. Term $R$ is the total resistance of the line, where $R / 2$ is concentrated at the nodes $k$ and $m$. The Bergeron model with losses is characterized by a constant resistance $R$, without the frequency effect in the longitudinal parameters. This line model is included in the most of the transient programs derived from the EMTP.

The inclusion of the frequency effect in the Bergeron circuit is possible using fitting techniques. In this context, the fitting procedure is basically the approximation of the impedance $Z(\omega)$ by a rational function $Z_{\text {fit }}(\omega)$. The residues and poles of the fitted impedance $Z_{\text {fit }}(\omega)$ are associated with a $R L$ circuit, which is the main step for inclusion of the frequency effect directly in the time domain.

The equivalent circuit that represents the impedance $Z(\omega)$ is described in Fig. 2.

The rational function $Z_{\text {fit }}(\omega)$ of the circuit in Fig. 2 is expressed as follows [9]:

$Z(\omega) \approx Z_{\text {fit }}(\omega)=R_{0}+j \omega L_{0}+\sum_{i=1}^{n} \frac{j \omega R_{i}}{\left(j \omega+R_{i} / L_{i}\right)}$

Term $\omega$ is the angular frequency. The resistance $R_{0}$ and the inductance $L_{0}$ are values for $\omega=0$. The number of $R L$ circuits in the equivalent circuit is a function of the frequency range considered in $Z_{\text {fit }}(\omega)$.

There are several fitting methods available in the technical literature for approximation of a general function by a rational function based on tabulated values (poles, zeros and residues). However, the vector fitting was firstly applied for TLM in reference [9], which describes an accurate algorithm for smooth and resonant responses with high order and wide frequency bands. This algorithm has been widely used for development of frequency-dependent line models in the time domain for simulation of electromagnetic transients in power systems [12-15].

The inclusion of the frequency effect in the Bergeron circuit consists in replacing the constant resistances that represent the line losses in Fig. 1, by the equivalent circuit described in Fig. 2. This procedure requires a new formulation of the Bergeron model that results in a system of differential equations with dimension proportional to the number RL blocks that composes circuit in Fig. 2 [15].

Substituting the resistance $R$ in Fig. 1 by the equivalent circuit obtained from $Z_{\text {fit }}(\omega)$, the frequency-dependent Bergeron circuit is restructured as follows: 


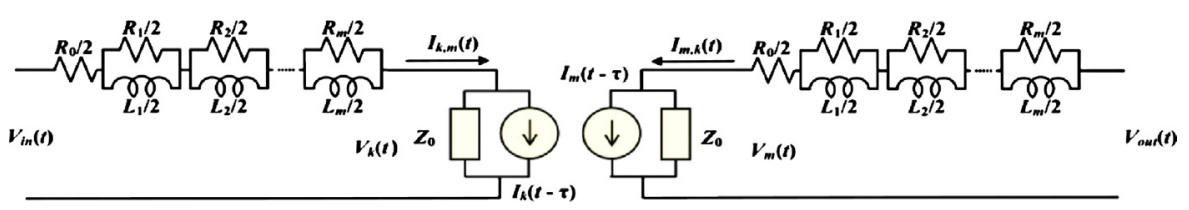

Fig. 3. Frequency-dependent Bergeron circuit in the time domain.

The frequency-dependent impedance is concentrated at the nodes $k$ and $m$ of the equivalent Bergeron circuit in Fig. 3, such as described for the Bergeron model with losses in Fig. 1. However, the constant resistance $R$ is replaced by the impedance $Z_{\text {fit }}(\omega)$, in (5), directly in the time domain. Another variations in the frequencydependent circuit in Fig. 3 is that the input and output voltages are indicated as $V_{\text {in }}(t)$ and $V_{\text {out }}(t)$. The voltages $V_{k}(t)$ and $V_{m}(t)$ are associated with the past-history current sources $I_{k}(t-\tau)$ and $I_{m}(t-\tau)$, respectively.

The number of $R L$ blocks depends on the type of electromagnetic transient to be analyzed. For an input signal composed of low frequencies, e.g. a switching operation, no more than three or four $R L$ circuits are necessary. Otherwise, for fast and impulsive transients, e.g. an atmospheric impulse, more than five $R L$ blocks are required to cover the entire range of frequencies that composes a steep-front wave [15].

Considering the frequency-dependent Bergeron circuit in Fig. 3, voltage equations on resistors and inductors of the node $k$ are expressed:

$\frac{R_{1}}{2}\left(i_{k 0}-i_{k 1}\right)=\frac{L_{1}}{2} \frac{\mathrm{d} i_{k 1}}{\mathrm{~d} t}$

$\frac{R_{2}}{2}\left(i_{k 0}-i_{k 2}\right)=\frac{L_{2}}{2} \frac{\mathrm{d} i_{k 2}}{\mathrm{~d} t}$

$\frac{R_{n}}{2}\left(i_{k 0}-i_{k n}\right)=\frac{L_{n}}{2} \frac{\mathrm{d} i_{k n}}{\mathrm{~d} t}$

Terms $i_{k 0}, i_{k 1}$ to $i_{k n}$ are the currents in the resistor $R_{0}$ and inductors $L_{1}$ to $L_{n}$, respectively. Following the Kirchhoff's current law, the currents through the resistors $R_{1}$ and $R_{n}$ are $\left(i_{k 0}-i_{k 1}\right)$ and $\left(i_{k 0}-i_{k n}\right)$, respectively. Thus, a general expression is formulated by association of the past-history current source $I_{k}(t-\tau)$ and the state current $i_{k 0}$ that is similar to the current $i_{k, m}(t)$.

$$
\begin{aligned}
& V_{\text {in }}-R_{0} i_{k 0}-R_{1}\left(i_{k 0}-i_{k 1}\right)-\ldots-R_{n}\left(i_{k 0}-i_{k n}\right)+Z_{0} I_{k}(t-\tau) \\
& \quad=Z_{0} i_{k 0}
\end{aligned}
$$

From expression (9), the currents and voltages of the frequencydependent Bergeron circuit in Fig. 3 can be calculated for a single-phase line. In Section 3, an optimized method for modal decoupling is described based on the use of two different modal transformation matrices. This modal decoupling method results in exact modal transformations, i.e., a multiphase representation of transmission lines without errors resulted from the successive modal transformations in the modeling and simulation processes [16].

\section{Multiconductor modeling of transmission lines using modal techniques}

The electromagnetic propagation characteristics of multiconductor transmission lines are dependent of the impedance and admittance matrices, $[\mathrm{Z}]$ and $[\mathrm{Y}]$, respectively. These matrices are frequency-dependent and expressed for an $\mathrm{N}$-phase transmission line as:

$[Z]=\left[\begin{array}{cccc}Z_{11} & Z_{12} & \cdots & Z_{1 N} \\ Z_{21} & Z_{22} & \cdots & Z_{2 N} \\ \vdots & \vdots & \ddots & \vdots \\ Z_{N 1} & Z_{N 2} & \cdots & Z_{N N}\end{array}\right]$
$[Y]=\left[\begin{array}{cccc}Y_{11} & Y_{12} & \cdots & Y_{1 N} \\ Y_{21} & Y_{22} & \cdots & Y_{2 N} \\ \vdots & \vdots & \ddots & \vdots \\ Y_{N 1} & Y_{N 2} & \cdots & Y_{N N}\end{array}\right]$

The explicit modeling of the mutual parameters in TLM represents a difficult issue because there is no consensus on the physical frequency-dependent modeling of the longitudinal parameters between two phases [17]. A usual tool to overcome this problem is by using modal decoupling techniques based on a modal transformation matrix.

The modal decoupling basically consists of the transformation of an $N$-phase transmission line in $N$ independent propagation modes. The modal transformation is carried out using a modal transformation matrix [8]:

$\left[\mathrm{Z}_{\mathrm{M}}\right]=\left[\mathrm{T}_{\mathrm{I}}\right]^{T}[\mathrm{Z}]\left[\mathrm{T}_{\mathrm{I}}\right]$

$\left[\mathrm{Y}_{\mathrm{M}}\right]=\left[\mathrm{T}_{\mathrm{I}}\right]^{-1}[\mathrm{Y}]\left[\mathrm{T}_{\mathrm{I}}\right]^{-T}$

As previously described, the transformation matrix $\left[\mathrm{T}_{\mathrm{I}}\right]$ is variable with the frequency. The row and columns of $\left[\mathrm{T}_{\mathrm{I}}\right]$ are calculated based on the eigenvalues and eigenvectors of the matrix product [Z][Y] using the Newton-Raphson method [8]. The modal transformation matrix for transmission line decoupling is approached in details in many technical literatures $[8,9,11,13,14]$ Terms $\left[T_{I}\right]^{-1}$ and $\left[\mathrm{T}_{\mathrm{I}}\right]^{T}$ are the inverse and transposed transformation matrices.

In (12) and (13), if the modal matrices $\left[\mathrm{Z}_{\mathrm{M}}\right]$ and $\left[\mathrm{Y}_{\mathrm{M}}\right]$ are ideally decoupled, their respective main diagonals are composed of modal impedances and admittances of the $N$ propagation modes whereas terms out of the main diagonal are null [8].

The relationships of currents and voltages in the modal and phase domains are also obtained using the same modal transformation matrix:

$\left[\mathrm{I}_{\mathrm{M}}\right]=\left[\mathrm{T}_{\mathrm{I}}\right]^{-1}[\mathrm{I}]$

$\left[\mathrm{V}_{\mathrm{M}}\right]=\left[\mathrm{T}_{\mathrm{I}}\right]^{T}[\mathrm{~V}]$

Terms [I] and [V] are vectors with $N$ currents and $N$ voltages of an $\mathrm{N}$-phase transmission line, respectively. Analogously, vectors $\left[\mathrm{I}_{\mathrm{M}}\right]$ and $\left[\mathrm{V}_{\mathrm{M}}\right]$ are composed of currents and voltages of the $N$ propagation modes.

Several line models available in the technical literature in TLM substitute the frequency-dependent matrix $\left[\mathrm{T}_{\mathrm{I}}\right]$ by a real and constant matrix in (12)-(15) $[11,14]$. Depending on the geometrical characteristics and structure of the transmission line, the approach using a real and constant matrix leads to inaccurate results in the modal decoupling. Thus, the proposed multiconductor representation is derived from a simple modification in the conventional 
modal decoupling in order to avoid eventual frequency- and timedomains errors. As a first step, the line decoupling for a three-phase line is accomplished using the exact frequency-dependent transformation matrix. From this procedure, the modal parameters can be completely decoupled from each other, without remaining mutual terms, as expressed in (16) and (17) [16].

$$
\begin{aligned}
& {\left[\mathrm{Z}_{\mathrm{M}}\right]=\left[\mathrm{T}_{\mathrm{I}}\right]^{T}[\mathrm{Z}]\left[\mathrm{T}_{\mathrm{I}}\right]=\left[\begin{array}{ccc}
Z_{\alpha} & 0 & 0 \\
0 & Z_{\beta} & 0 \\
0 & 0 & Z_{0}
\end{array}\right]} \\
& {\left[\mathrm{Y}_{\mathrm{M}}\right]=\left[\mathrm{T}_{\mathrm{I}}\right]^{-1}[\mathrm{Y}]\left[\mathrm{T}_{\mathrm{I}}\right]^{-T}=\left[\begin{array}{ccc}
Y_{\alpha} & 0 & 0 \\
0 & Y_{\beta} & 0 \\
0 & 0 & Y_{0}
\end{array}\right]}
\end{aligned}
$$

Since the line was totally decoupled from (12) and (13), there are no errors in the line modeling and in the currents and voltages in (14) and (15). The exact propagation modes $\alpha, \beta$ and 0 can be represented as three independent single-phase lines and modeled by the frequency-dependent Bergeron circuit in Fig. 3. Thus, the exact frequency-dependent matrix cannot be used in (14) and (15) because the propagation modes were prior modeled direct in the time domain. In this case, the approach using a real and constant transformation matrix is necessary, such as the Clarke's matrix that is used in several well-established line models available in the TLM literature [11,14]. The Clarke's matrix is a validity approach for transposed transmission lines or for transmission lines with a vertical symmetric plane Eqs. (14) and (15) can be reformulated with the Clarke's matrix as follows [16]:

$$
\begin{aligned}
& {\left[\mathrm{I}_{\mathrm{M}}\right]=\left[\mathrm{T}_{\mathrm{C}}\right]^{-1}[\mathrm{I}]=\left[\begin{array}{lll}
I_{\alpha} & I_{\beta} & I_{0}
\end{array}\right]^{T}} \\
& {\left[\mathrm{~V}_{\mathrm{M}}\right]=\left[\mathrm{T}_{\mathrm{C}}\right]^{T}[\mathrm{~V}]=\left[\begin{array}{lll}
V_{\alpha} & V_{\beta} & V_{0}
\end{array}\right]^{T}}
\end{aligned}
$$

The transformation matrix $\left[\mathrm{T}_{\mathrm{C}}\right]$ is the Clarke's matrix.

The modal procedure using two transformation matrices, the exact frequency-dependent matrix in the parameters decoupling and the Clarke's matrix for time-domain analyses, shows to be an efficient method to eliminate eventual errors resulted from the modal decoupling in multiconductor line modeling [16]. Other line models, based on modal decoupling techniques, have considered the Clarke's matrix for the entire decoupling procedure $[8,11,14]$. This approach implies inaccuracies in time-domain simulations of the currents and voltages of the line because the propagation modes are modeled from inaccurate parameters.

\section{Three-phase line modeling from the frequency-dependent Bergeron approach}

The propagation modes $\alpha, \beta$ and 0 , in (16) and (17), can be modeled as three single-phase lines using the frequencydependent Bergeron circuit in Fig. 3. Thus, (6)-(9) are expressed in the modal domain:

$$
\begin{aligned}
& \frac{R_{M 1}}{2}\left(i_{M k 0}-i_{M k 1}\right)=\frac{L_{M 1}}{2} \frac{\mathrm{d} i_{M k 1}}{\mathrm{~d} t} \\
& \frac{R_{M 2}}{2}\left(i_{M k 0}-i_{M k 2}\right)=\frac{L_{M 2}}{2} \frac{\mathrm{d} i_{M k 2}}{\mathrm{~d} t} \\
& \frac{R_{M n}}{2}\left(i_{M k 0}-i_{M k n}\right)=\frac{L_{M n}}{2} \frac{\mathrm{d} i_{M k n}}{\mathrm{~d} t}
\end{aligned}
$$

In (20)-(22), terms $i_{\mathrm{M} k 0}, i_{\mathrm{M} k 1}$ and $i_{\mathrm{M} k n}$ are the currents in the resistor $R_{M 0}$ and inductors $L_{M 1}$ to $L_{M n}$, respectively. The subscript $M$ indicates the propagation modes $\alpha, \beta$ and 0 . Thus, the system of $n$ differential equations, represented in (20)-(22), is in the modal domain and the $R_{M}$ and $L_{M}$ parameters are lumped elements obtained from the fitted function $Z_{M \text { fit }}$ based on $Z_{M}(\omega)$, analogously as expressed in (5). Thus, the system described generically in (20)-(22) represents three independent systems (for $M=\alpha, \beta, 0$ ) that are solved in parallel.

From the same way as in (6)-(8), the current in the resistors $R_{\mathrm{M} 1}$ and $R_{M n}$ are $\left(i_{M k 0}-i_{M k 1}\right)$ and $\left(i_{M k 0}-i_{M k n}\right)$, respectively. Thus, the general expression is developed associating the past-history current source $I_{M k}(t-\tau)$ to the state current $i_{M k 0}$ that is equivalent to the current $i_{M k, m}(t)$ in (25), analogously as in Fig. 3 and in the general expression in (9).

$$
\begin{aligned}
& V_{M i n}-R_{M 0} i_{M k 0}-R_{M 1}\left(i_{M k 0}-i_{M k 1}\right)-\ldots-R_{M n}\left(i_{M k 0}-i_{M k n}\right) \\
& +Z_{M 0} I_{M k}(t-\tau)=Z_{M 0} i_{M k 0}
\end{aligned}
$$

Term $Z_{\mathrm{M} 0}$ is the characteristic impedance of the generic propagation mode $M$.

The first-order system of differential equations, expressed in (20)-(23), can be expressed individually as state equations for each propagation mode [15]:

$$
\begin{aligned}
& {\left[\dot{\mathrm{I}}_{\alpha k}\right]=\left[\mathrm{A}_{\alpha k}\right]\left[\mathrm{I}_{\alpha k}\right]+\left[\mathrm{B}_{\alpha k}\right]\left[\mathrm{S}_{\alpha}\right]} \\
& {\left[\dot{\mathrm{I}}_{\beta k}\right]=\left[\mathrm{A}_{\beta k}\right]\left[\mathrm{I}_{\beta k}\right]+\left[\mathrm{B}_{\beta k}\right]\left[\mathrm{S}_{\beta}\right]} \\
& {\left[\dot{\mathrm{I}}_{0 k}\right]=\left[\mathrm{A}_{0 k}\right]\left[\mathrm{I}_{0 k}\right]+\left[\mathrm{B}_{0 k}\right]\left[\mathrm{S}_{0}\right]}
\end{aligned}
$$

Considering the index $M=\alpha, \beta 0$; the state-space systems in (24) are expressed in general terms:

$\left[\dot{\mathrm{I}}_{M k}\right]=\left[\mathrm{A}_{M k}\right]\left[\mathrm{I}_{M k}\right]+\left[\mathrm{B}_{M k}\right]\left[\mathrm{S}_{M}\right]$

Vector $\left[\mathrm{I}_{M k}\right]$ is composed of currents in the inductors $L_{M 1}$ to $L_{M n}$ :

$\left[\mathrm{I}_{M k}\right]^{T}=\left[\begin{array}{llll}i_{M k 1} & i_{M k 2} & \ldots & i_{M k n}\end{array}\right]$

The derivate form of the current vector in (25) and (26) is expressed as:

$\left[\dot{\mathrm{I}}_{M k}\right]^{T}=\left[\begin{array}{llll}\frac{\mathrm{d} i_{M k 1}}{\mathrm{~d} t} & \frac{\mathrm{d} i_{M k 2}}{\mathrm{~d} t} & \ldots & \frac{\mathrm{d} i_{M k n}}{\mathrm{~d} t}\end{array}\right]$

The state matrix $\left[\mathrm{A}_{M k}\right]$ is constant and expressed as a function of $R_{M}$ and $L_{M}$ values, obtained from the fitting procedure, and also the characteristic impedance $Z_{M 0}$, calculated based on the $L_{M}$ and $C_{M}$ parameters for direct current, as generically described in (3). Matrix $\left[\mathrm{A}_{M k}\right]$ is expressed as:

$$
\left[\mathrm{A}_{M k}\right]=\left[\begin{array}{llll}
\frac{R_{M 1}}{L_{M 1}}\left(-1+\frac{R_{M 1}}{Z_{M 0}+\sum_{n}^{i=1} R_{M i}}\right) & \frac{R_{M 1}}{L_{M 1}}\left(\frac{R_{M 2}}{Z_{M 0}+\sum_{n}^{i=1} R_{M i}}\right) & \ldots & \frac{R_{M 1}}{L_{M 1}}\left(\frac{R_{M n}}{Z_{M 0}+\sum_{n}^{i=1} R_{M i}}\right) \\
\frac{R_{M 2}}{L_{M 2}}\left(\frac{R_{M 1}}{Z_{M 0}+\sum_{i=1}^{n} R_{M i}}\right) & \frac{R_{M 2}}{L_{M 2}}\left(-1+\frac{R_{M 2}}{Z_{M 0}+\sum_{i=1}^{n} R_{M i}}\right) & \ldots & \frac{R_{M 2}}{L_{2}}\left(\frac{R_{M n}}{Z_{M 0}+\sum_{i=1}^{n} R_{M i}}\right) \\
\vdots & \vdots & \ddots & \vdots \\
\frac{R_{M n}}{L_{M n}}\left(\frac{R_{M 1}}{Z_{M 0}+\sum_{i=1}^{n} R_{M i}}\right) & \frac{R_{M n}}{L_{M n}}\left(\frac{R_{M 2}}{Z_{M 0}+\sum_{i=1}^{n} R_{M i}}\right) & \ldots & \frac{R_{M n}}{L_{M n}}\left(-1+\frac{R_{M n}}{Z_{M 0}+\sum_{i=1}^{n} R_{M i}}\right)
\end{array}\right]
$$


Matrix $\left[\mathrm{B}_{M k}\right]$ is also expressed based on the characteristic impedance $Z_{M 0}$ and the $R_{M}$ and $L_{M}$ values. Matrix [ $\mathrm{B}_{M k}$ ] has dimension $n$ per 2 and is expressed as follows:

$\left[\mathrm{B}_{M k}\right]=\left[\begin{array}{ll}\frac{R_{M 1}}{L_{M 1}}\left(\frac{1}{Z_{M 0}+\sum_{i=1}^{n} R_{M i}}\right) & \frac{R_{M 1}}{L_{M 1}}\left(\frac{Z_{M 0}}{Z_{M 0}+\sum_{i=1}^{n} R_{M i}}\right) \\ \frac{R_{M 2}}{L_{M 2}}\left(\frac{1}{Z_{M 0}+\sum_{i=1}^{n} R_{M i}}\right) & \frac{R_{M 2}}{L_{M 2}}\left(\frac{Z_{M 0}}{Z_{M 0}+\sum_{i=1}^{n} R_{M i}}\right) \\ \vdots & \vdots \\ \frac{R_{M n}}{L_{M n}}\left(\frac{1}{Z_{M 0}+\sum_{i=1}^{n} R_{M i}}\right) & \frac{R_{M n}}{L_{M n}}\left(\frac{Z_{M 0}}{Z_{M 0}+\sum_{i=1}^{n} R_{M i}}\right)\end{array}\right]$

Eq. (30) expresses the modal vector $\left[\mathrm{S}_{\mathrm{M}}\right]$ that is composed of the voltage source $V_{M s}$, represented by a time-variable input signal $V_{\text {Min }}(t)$, and the historical current source $I_{M k}(t-\tau)$.

$\left[S_{\mathrm{M}}\right]=\left[\begin{array}{c}V_{\mathrm{Min}}(t) \\ I_{M k}(t-\tau)\end{array}\right]$

The input currents and voltages at the sending end of the threephase line should be converted to the modal domain. Considering a balanced three-phase source (e.g. a synchronous generator) connected at the sending end of the line, the phase voltages at the three terminals should be converted to the modal domain using (18) and (19). From this procedure, the input voltage $V_{\text {Min }}(t)$ at the sending terminal of the modes $\alpha, \beta$ and 0 are obtained and the historical current source $I_{M k}(t-\tau)$ is calculated direct in the modal domain.

From some algebraic adaptations in the state-space formulation in (25) and based on the circuit in Fig. 3, the total current $I_{k, m}(t)$ and voltage $V_{k}(t)$ (in Fig. 3) are expressed as $I_{M k, m}(t)$ and $V_{M k}(t)$, respectively, in the modal domain [15]:

$$
\begin{aligned}
I_{M k, m}(t)= & \sum_{q=1}^{n}\left(\frac{R_{M q} i_{M k q}}{Z_{M 0}+\sum_{n}^{i=1} R_{M i}}\right)+\frac{1}{Z_{M 0}+\sum_{n}^{i=1} R_{M i}} V_{M i n} \\
& +\frac{Z_{M 0}}{Z_{M 0}+\sum_{n}^{i=1} R_{M i}} I_{M k}(t-\tau)
\end{aligned}
$$

$V_{M k}(t)=V_{\mathrm{Min}}(t)-\sum_{i=1}^{n} R_{M i} i_{M k i}$

The formulation for the node $m$ is similar to the development of the node $k$ because the parameters of the frequency-dependent circuit are the same for both sides of the equivalent circuit in Fig. 3. Considering a generic load, represented by an impedance $Z_{L}$ at node $m$, the state matrix $\left[A_{M m}\right]$ is expressed as: power element, such as a variable load or even a protection component (metal-oxide surge arrester or a switch). By contrast, for line models in the frequency domain, the inclusion of other non-linear elements is not a simple procedure because most power components are not usually approached in the frequency domain $[13,14]$.

The vector with the modal currents in $L_{\mathrm{M} 1}$ to $L_{\mathrm{M} n}$ can be renamed as $\left[\mathrm{I}_{\mathrm{M} m}\right]$. However, as the $k$ and $m$ sections of the Bergeron circuit are similar:

$$
\begin{aligned}
& {\left[\mathrm{I}_{\mathrm{M} m}\right]^{T}=\left[\mathrm{I}_{\mathrm{M} k}\right]^{T}=\left[\begin{array}{llll}
i_{\mathrm{M} k 1} & i_{\mathrm{M} k 2} & \ldots & i_{\mathrm{M} k n}
\end{array}\right]} \\
& \text { and } \\
& {\left[\dot{\mathrm{I}}_{M m}\right]^{T}=\left[\dot{\mathrm{I}}_{M k}\right]^{T}=\left[\begin{array}{llll}
\frac{\mathrm{d} i_{M k 1}}{\mathrm{~d} t} & \frac{\mathrm{d} i_{M k 2}}{\mathrm{~d} t} & \ldots & \frac{\mathrm{d} i_{M k n}}{\mathrm{~d} t}
\end{array}\right]}
\end{aligned}
$$

As shown in (34)-(35), vector $\left[\mathrm{I}_{M m}\right]$ can be expressed only in terms of $i_{M k}$.

Terms $\left[\mathrm{B}_{\mathrm{M}}\right]$ and $\left[\mathrm{S}_{\mathrm{M}}\right]$ in (25) are also modified. Matrix $\left[\mathrm{B}_{\mathrm{M}}\right]$ is reformulated as a vector composed of a single column:

$\left[\mathrm{B}_{\mathrm{M} m}\right]=\left[\begin{array}{l}\frac{R_{M 1}}{L_{M 1}}\left(\frac{Z_{M 0}}{Z_{M 0}+Z_{L}+\sum_{i=1}^{n} R_{M i}}\right) \\ \frac{R_{M 2}}{L_{M 2}}\left(\frac{Z_{M 0}}{Z_{M 0}+Z_{L}+\sum_{i=1}^{n} R_{M i}}\right) \\ \vdots \\ \frac{R_{M m}}{L_{M m}}\left(\frac{Z_{M 0}}{Z_{M 0}+Z_{L}+\sum_{i=1}^{n} R_{M i}}\right)\end{array}\right]$

Vector $\left[S_{M}\right]$ is substituted by the past-history current source $I_{m}(t-\tau)$ from the node $m$. Thus, the state equation in (25) can be rewritten from the current source in node $m$ of the equivalent Bergeron circuit in Fig. 3:

$\left[\dot{\mathrm{I}}_{M k}\right]=\left[\mathrm{A}_{M n}\right]\left[\mathrm{I}_{M k}\right]+\left[\mathrm{B}_{M n}\right] I_{M m}(t-\tau)$

Analogously to (31) and (32), the current $I_{M m, k}(t)$ and the voltage $V_{M m}(t)$ are analytically expressed:

$I_{M m, k}(t)=\frac{1}{Z_{M 0}+Z_{L}+\sum_{n}^{i=1} R_{M i}}\left[\sum_{q=1}^{n} R_{M q} i_{M k q}+Z_{M 0} I_{M m}(t-\tau)\right]$

$V_{M m}(t)=V_{M o u t}(t)-\sum_{i=1}^{n} R_{M i} i_{M k i}$

The output voltage $V_{\text {Mout }}(t)$ is expressed as:

$V_{\text {Mout }}(t)=-I_{M m, k}(t) Z_{L}$

The state matrices are obtained from the fitted $R$ and $L$ parameters in the modal domain for $M=\alpha, \beta, 0$; the first-order systems,

$$
\left[A_{\mathrm{M} m}\right]=\left[\begin{array}{llll}
\frac{R_{M 1}}{L_{M 1}}\left(-1+\frac{R_{M 1}}{Z_{M 0}+Z_{L}+\sum_{i=1}^{n} R_{M i}}\right) & \frac{R_{M 1}}{L_{M 1}}\left(\frac{R_{M 2}}{Z_{M 0}+Z_{L}+\sum_{i=1}^{n} R_{M i}}\right) & \cdots & \frac{R_{M 1}}{L_{M 1}}\left(\frac{R_{M n}}{Z_{M 0}+Z_{L}+\sum_{i=1}^{n} R_{M i}}\right) \\
\frac{R_{M 2}}{L_{M 2}}\left(\frac{R_{M 1}}{Z_{M 0}+Z_{L}+\sum_{i=1}^{n} R_{M i}}\right) & \frac{R_{M 2}}{L_{M 2}}\left(-1+\frac{R_{M 2}}{Z_{M 0}+Z_{L}+\sum_{i=1}^{n} R_{M i}}\right) & \cdots & \frac{R_{M 2}}{L_{M 2}}\left(\frac{R_{M n}}{Z_{M 0}+Z_{L}+\sum_{i=1}^{n} R_{M i}}\right) \\
\vdots & \vdots & \ddots & \vdots \\
\frac{R_{M n}}{L_{M n}}\left(\frac{R_{M 1}}{Z_{M 0}+Z_{L}+\sum_{i=1}^{n} R_{M i}}\right) & \frac{R_{M n}}{L_{M n}}\left(\frac{R_{M 2}}{Z_{M 0}+Z_{L}+\sum_{i=1}^{n} R_{M i}}\right) & \cdots & \frac{R_{M n}}{L_{M n}}\left(-1+\frac{R_{M n}}{Z_{M 0}+Z_{L}+\sum_{i=1}^{n} R_{(M) i}}\right)
\end{array}\right]
$$

One of the main advantages in the time-domain modeling is the simple inclusion of time-variable elements in simulations. Thus, the load $Z_{L}$ can be modeled as constant impedance or a time-variable representing each propagation mode, are solved using numerical or analytical integration methods $[14,15]$. 


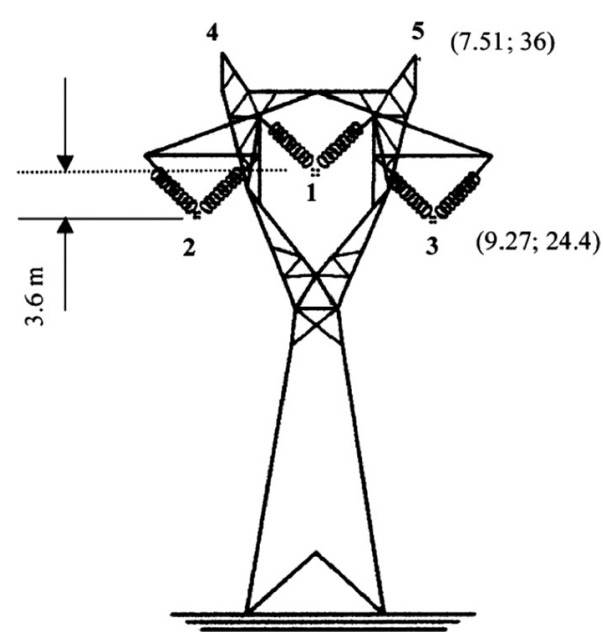

Fig. 4. Three-phase transmission line with a single circuit.

The same mode-phase relationships expressed in (20) and (21) are used to obtain the phase values of $I_{m, k}(t)$ and $V_{m}(t)$ for the three phases at the receiving end of the line, based on the calculated modal values of $I_{M m, k}(t)$ and $V_{M m}(t)$. The phase values are represented by the vectors $[\mathrm{I}]$ and $[\mathrm{V}]$ whereas $\left[\mathrm{I}_{\mathrm{M}}\right]$ and $\left[\mathrm{V}_{\mathrm{M}}\right]$ are vectors with modal currents and voltages, respectively, as described in (18) and (19).

\section{Validation of the proposed three-phase line model}

The proposed multiconductor line model is validated based on results obtained from the well-established Universal Line Model (ULM). This reference model is developed in the frequency domain based on the two-port equations of the line. The time-domain simulations are obtained from inverse transform. The ULM is a very accurate model for transient simulations without non-linear and time-variable elements in the system [13].

Initially, a conventional $440-\mathrm{kV}$ line section with length of $300 \mathrm{~km}$ is modeled using the two line models: the proposed Bergeron model and the ULM. The geometrical characteristics of the line/tower are described in Fig. 4. Each phase consists of a bundle with four Grosbeak sub-conductors and the ground wires are EHWS-3/8“. A soil resistivity of $1000 \Omega \mathrm{m}$ was considered for calculation of the line impedances.

For the proposed line model, the fitted parameters are up to $1 \mathrm{MHz}$, as described in details in the appendix. The systems of state equations in (24), representing the proposed Bergeron model, are solved using the well-established trapezoidal rule $[12,14,15]$. The transmission line has the sending end connected to a synchronous generator and the receiving end connected to a three-phase load of 60 MVA with power factor of 0.98 inductive. From the initial simulation time $t=0$, a three-phase switch is closed at the sending end of the line, connecting the synchronous generator to the line. The voltages at the load terminal were simulated using the two models, as described in Fig. 5.

The solid and dotted curves in Fig. 5 are the results obtained from the ULM and the proposed line model, respectively. The voltages at the receiving ends of the three phases are described for a time window of $20 \mathrm{~ms}$. The magnitude and shape of the voltages obtained from the two line models are similar in the transient and steady states. Only a small phase shift is observed during the first $5 \mathrm{~ms}$ of simulation, probably because of eventual truncation and aliasing errors of the inverse transform in the ULM. The simulation times of the two computational procedures (proposed model and ULM) are no greater than $5 \mathrm{~s}$.

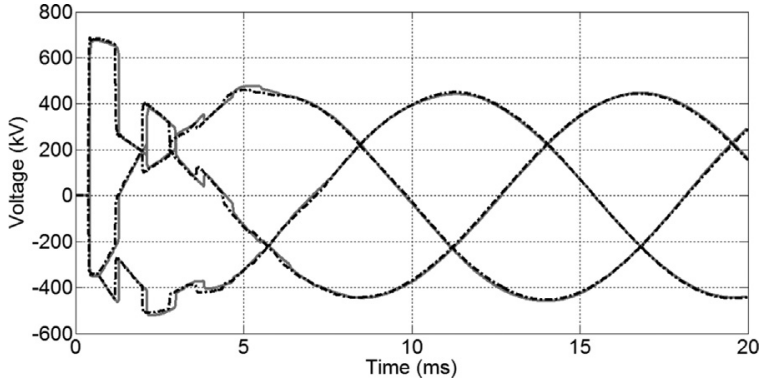

Fig. 5. Voltages at the receiving end of the transmission line: ULM (solid curves) and proposed line model (dotted curves).

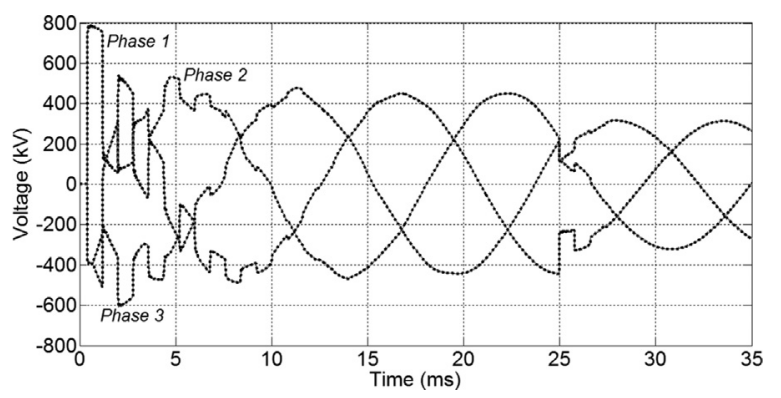

Fig. 6. Transient voltages simulated at the receiving end of the transmission line with a variable load profile: proposed line model (dotted curves) and J. Marti model (solid curves).

\section{Simulation of time-variable elements using the proposed line model}

The proposed line model is able to simulate several line configurations considering non-linear and time-variable conditions. Based on the same $440-\mathrm{kV}$ transmission line approached in the last section, two distinct time-variable situations are simulated: a variable load profile and a three-phase fault.

In the first case, a synchronous generator is connected to the sending end of the line at the initial time. In sequence, after the transient state resulted from the line switching, an abrupt load variation occurs at the receiving end of the line, resulting a new transient state in the system. This first case is simulated using the proposed line model and the time-domain model of J. Marti (available in the EMTP) in order to validate transient simulations including time-variable conditions. The voltage and current transients are described in Figs. 6 and 7, respectively.

An initial load with an apparent power of 150 MVA and power factor of 0.98 is connected to the receiving end of the line. After $25 \mathrm{~ms}$ of simulation, the load impedance is abruptly varied. This non-linear behavior results in voltage and current transients that

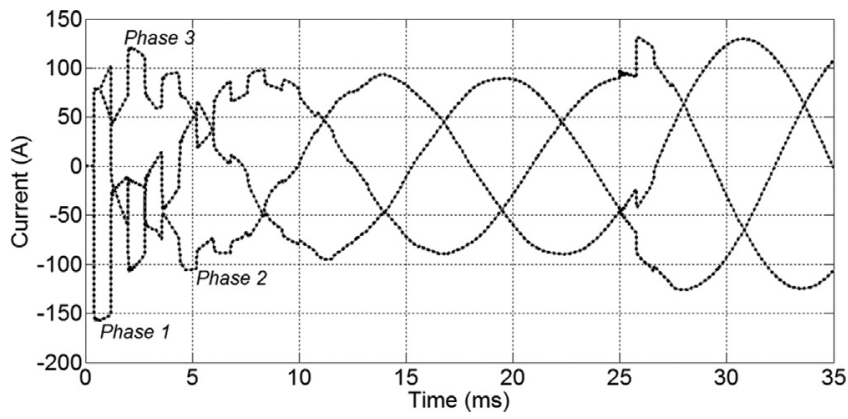

Fig. 7. Transient currents simulated at the receiving end of the transmission line with a variable load profile: proposed line model (dotted curves) and J. Marti model (solid curves). 


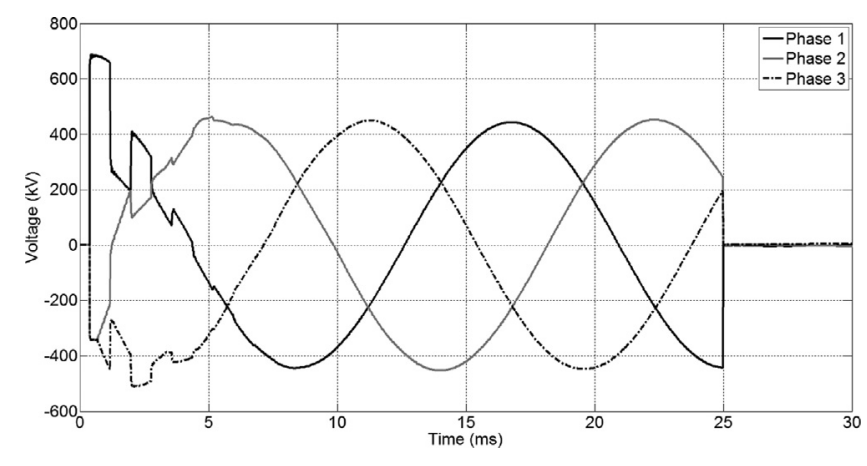

Fig. 8. Transient voltages simulated using the proposed model at the receiving end of the transmission line during a switching followed by a three-phase fault.

are observed in Figs. 6 and 7, respectively. In fact, this non-linear behavior and similar results can be obtained from an abrupt variation in the load characteristics and also from the tap changing of power transform connected at the line terminals. Though power systems are characterized by a time-variable dynamic, the nonlinear elements that represent this dynamic behavior are not easily modeled using frequency-domain models, as in the ULM. By contrast, the proposed line model is completely developed in the time domain, enabling a flexible inclusion of time-variable elements during simulations. This characteristic can be proved comparing the results obtained from the proposed line model (dotted curves) and results simulated using the well-established line model of $\mathrm{J}$. Marti (solid curve). Curves obtained from the proposed and J. Marti models are practically overlapped, validating the proposed model for simulations including non-linear and time-variable cases. The relative errors of simulations carried out using the two models are no greater than $0.001 \%$, i.e., the results are practically similar. An important additional observation in this first simulation is that the current values in Fig. 7 have inverse signals when compared to the transient voltages in Fig. 6 because of a simple notation in the signal conventions using the Bergeron model. It was assumed that the current flowing from the receiving ends of the line to the load is negative. However, this convention could be easily modified in the line modeling and simulations.

In the second case, a three-phase switching is simulated at the sending end of the line followed by a three-phase fault. This type of fault represents a very critical situation that in the most of the cases the entire transmission system is deactivated by the primary and secondary protection of the line and substations. Initially, a synchronous generator is connected at the sending end of the transmission line. After $25 \mathrm{~ms}$ from the line switching, a three-phase fault occurrence is simulated at the receiving end of line. Fig. 8 shows the voltage profile at the load terminal of the line for the three phases:

The voltage transients and residual voltages on the three-phase load during the fault occurrence in Fig. 8 are observed in details in Fig. 9, extending a time window from $25 \mathrm{~ms}$ up to $50 \mathrm{~ms}$.

The voltage transients, resulted from successive wave reflections between the line terminals, are observed from $25 \mathrm{~ms}$ up to $30 \mathrm{~ms}$. Residual voltage peaks of no more than $6 \mathrm{kV}$ are observed after $30 \mathrm{~ms}$ of simulation, as shown in Fig. 9.

The current transients at the load terminal are described in Fig. 10.

In the time interval from $25 \mathrm{~ms}$ to $28 \mathrm{~ms}$, a sequence of current wave reflections are observed at the receiving end of the line. From $30 \mathrm{~ms}$ of simulation, the currents at the load terminal presented peaks of $1.5 \mathrm{kA}$ up to $2 \mathrm{kA}$. As additional information, the execution times, during simulations using the two models, are less than $5 \mathrm{~s}$.

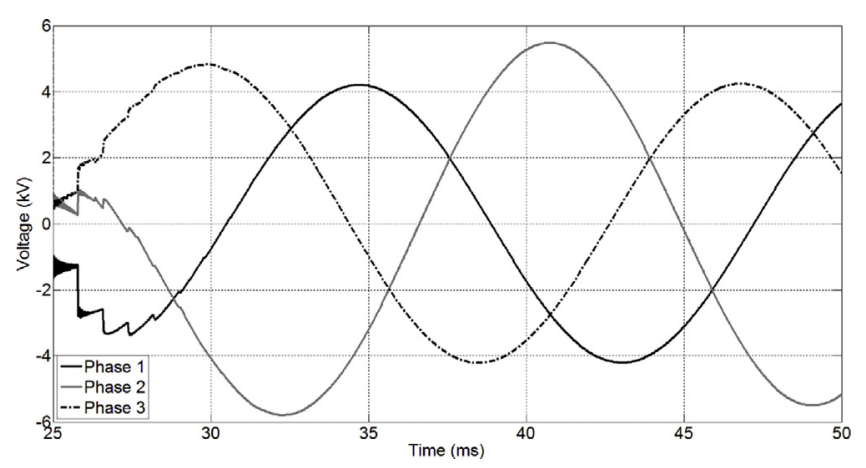

Fig. 9. Transient voltages simulated using the proposed model at the receiving end of the transmission line during the three-phase fault.

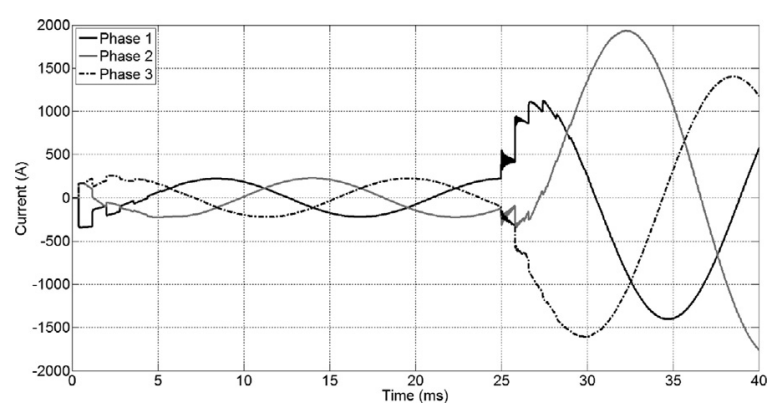

Fig. 10. Transient currents simulated using the proposed model at the receiving end of the transmission line during a switching followed by a three-phase fault.

\section{Conclusions}

A frequency-dependent line model based on the method of characteristics was proposed for a multiconductor transmission line. The proposed line model was developed from the Bergeron equivalent circuit and using fitting techniques for inclusion of the frequency effect directly in the time domain. An accurate modal decoupling technique was applied for the multiphase modeling, resulting in ideal line decoupling in exact propagation modes, i.e., without remaining mutual terms in the matrices $\left[Z_{M}\right]$ and $\left[Y_{M}\right]$.

The proposed line model was validated by comparison with results obtained from the well-established Universal Line Model-ULM. In sequence, the proposed model was tested for several time-variable conditions as: switching, variable load and a three-phase fault. The electromagnetic transients simulated including these time-variable elements show to be accurate and in accordance with the expected results for the modeled transmission line.

The accuracy of the proposed model depends on two distinct steps in the line modeling. The first step is the line decoupling into the propagation modes. The proposed decoupling technique has presented a great performance in TLM, as described in previous references. An untransposed three-phase line can be decoupled into three exact propagation modes using the exact frequency-dependent transformation matrix whereas the timedomain modeling/simulation process is carried out based on the approach using the Clarke's matrix. This line modeling technique results in three exact propagation modes and no inaccuracies in the time-domain simulations. The second step is the impedance fitting of the propagation modes, which were fitted up to $1 \mathrm{MHz}$ using seven pairs of residues and poles of $Z_{\text {fit }}(\omega)$, as described in the appendix. The accuracy of the simulations is directly dependent on the fitting technique applied in the line modeling. The fitting algorithm applied in the proposed line model is well established in the technical literature on TLM and proved to be very robust 

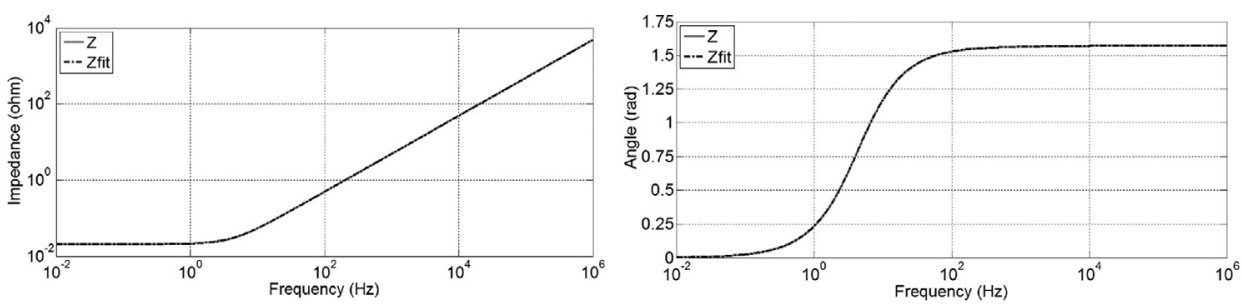

(a)

Fig. 11. Modulus (a) and angle (b) of the impedances $Z(\omega)$ and $Z_{\text {fit }}(\omega)$ of the propagation mode $\alpha$.
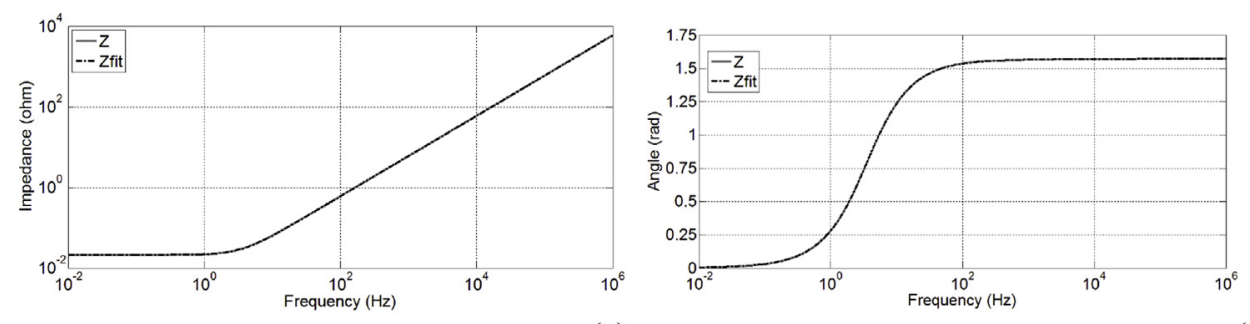

$(a)$

Fig. 12. Modulus (a) and angle (b) of the impedances $Z(\omega)$ and $Z_{\mathrm{fit}}(\omega)$ of the propagation mode $\beta$.
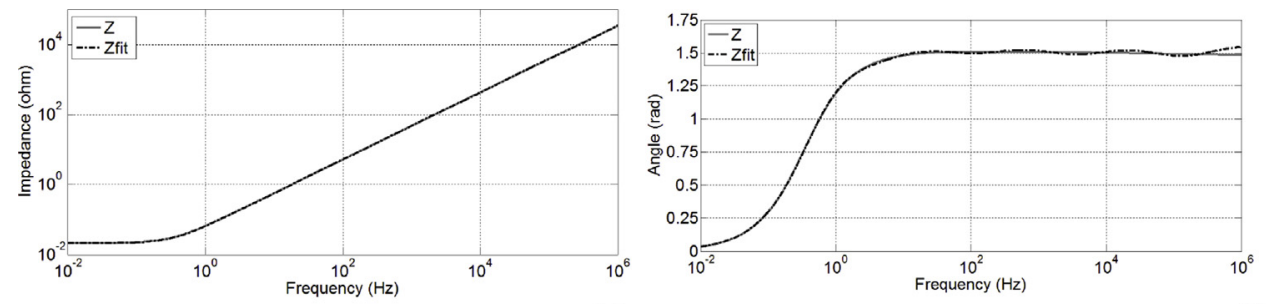

(a)

Fig. 13. Modulus (a) and angle (b) of the impedances $Z(\omega)$ and $Z_{\text {fit }}(\omega)$ of the propagation mode 0 .

if an adequate number of residues and poles are considered to synthesize the fitted impedance $Z_{\text {fit }}(\omega)$.

\section{Acknowledgment}

This research received financial support of the São Paulo Research Foundation-FAPESP (Procs. 13/00974-5 and 14/17051$0)$.

\section{Appendix A. Appendix}

The impedances $Z(\omega)$ and $Z_{\text {fit }}(\omega)$ were calculated and fitted, respectively, for the propagation modes $\alpha, \beta$ and 0 for a frequency range up to $1 \mathrm{MHz}$ and considering seven pairs of residues and poles. The modeling and simulation process were carried out using the commercial software Matlab ${ }^{\mathrm{TM}}$. The modulus and angles of $Z(\omega)$ and $Z_{\text {fit }}(\omega)$ for the three independent modes are described in Figs. 11-13.

Figs. 11-13 showed that $Z(\omega)$ and $Z_{\text {fit }}(\omega)$ are practically overlapped for the three propagation modes. Only a few oscillations were observed in the angle of $Z_{\mathrm{fit}}(\omega)$ of the propagation mode 0 for frequencies higher than $100 \mathrm{~Hz}$ (Fig. 13b). These oscillations could be easily eliminated increasing the number of poles and zeros in $Z_{\text {fit }}(\omega)$, however, these inaccuracies do not represent significant errors in the time-domain simulations.

The resistances and inductances of the equivalent circuit in Fig. 2, obtained from the vector fitting algorithm, are described in Tables 1 and 2, respectively.
Table 1

Resistance values for the modal impedance fitting.

\begin{tabular}{lccc}
\hline & Mode $\alpha(\Omega)$ & Mode $\beta(\Omega)$ & Mode $\boldsymbol{O}(\Omega)$ \\
\hline$R_{0}$ & 2.0831 & 2.0831 & 2.0861 \\
$R_{1}$ & 71.2035 & 71.3166 & 86510.9063 \\
$R_{2}$ & 19.4688 & 19.4799 & 2263.9523 \\
$R_{3}$ & 7.3683 & 7.3706 & 69.9995 \\
$R_{4}$ & 3.2667 & 3.2672 & 2.3584 \\
$R_{5}$ & 2.1433 & 2.1434 & 0.0978 \\
$R_{6}$ & $1.043 \times 10^{-12}$ & $1.044 \times 10^{-12}$ & $343.6960 \times 10^{-9}$ \\
\hline
\end{tabular}

Table 2

Inductance values for the modal impedance fitting

\begin{tabular}{lccc}
\hline & Mode $\alpha(\mathrm{mH})$ & Mode $\beta(\mathrm{mH})$ & Mode $0(\mathrm{mH})$ \\
\hline$L_{0}$ & $761.6470 \times 10^{-3}$ & $933.5407 \times 10^{-3}$ & $5412.0362 \times 10^{-3}$ \\
$L_{1}$ & $408.3023 \times 10^{-6}$ & $408.3302 \times 10^{-6}$ & $1089.2526 \times 10^{-3}$ \\
$L_{2}$ & $820.2195 \times 10^{-6}$ & $820.1767 \times 10^{-6}$ & $1052.8664 \times 10^{-3}$ \\
$L_{3}$ & $1522.1062 \times 10^{-6}$ & $1522.1401 \times 10^{-6}$ & $1024.7524 \times 10^{-3}$ \\
$L_{4}$ & $2633.7367 \times 10^{-6}$ & $2633.9204 \times 10^{-6}$ & $975.7775 \times 10^{-3}$ \\
$L_{5}$ & $6944.4064 \times 10^{-6}$ & $6944.4932 \times 10^{-6}$ & $909.8750 \times 10^{-3}$ \\
$L_{6}$ & $324.097 \times 10^{-9}$ & $324.232 \times 10^{-9}$ & $339.1479 \times 10^{-3}$
\end{tabular}

\section{References}

[1] H.F. Branin, Computer methods of network analysis, Proc. IEEE 55 (1967) 1787-1801.

[2] A. Budner, Introduction of frequency-dependent line parameters into an electromagnetic transients program, IEEE Trans. Power Appar. Syst. 89 (1) (1970) 88-97.

[3] J.K. Snelson, Propagation of travelling waves in transmission lines: frequency dependent parameters, IEEE Trans. Power Appar. Syst. PAS-91 (1972) 85-91. 
[4] H.W. Dommel, Digital computer solution of electromagnetic transients in single- and multi-phase networks, IEEE Trans. Power Appar. Syst. 88 (4) (1969) 388-399.

[5] R.M. Nelms, G.B. Sheble, S.R. Newton, L.L. Grigsby, Using a personal computer to teach power system transients, IEEE Trans. Power Syst. 4 (3) (1989) 1293-1297.

[6] H.W. Dommel, Electromagnetic Transient Program Reference Manual (EMTP Theory Book), Dept. Electrical Engineering, University of British Columbia, Vancouver, Canada, 1989.

[7] J.R. Marti, Accurate Modelling of Frequency-Dependent Transmission Lines in Electromagnetic Transient Simulations, IEEE Trans. Power Appar. Syst. PAS-101 (1 (Jan)) (1982) 147-157.

[8] L.M. Wedephol, H.V. Nguyen, G.D. Irwin, Frequency-dependent transformation matrices for untransposed transmission lines using Newton-Raphson method, IEEE Trans. Power Syst. 11 (3) (1996) 1538-1546.

[9] B. Gustavsen, A. Semlyen, Combined phase and modal calculation of transmission line transients based on vector fitting, IEEE Trans. Power Deliv. 13 (2) (1998) 596-604.

[10] A. Ametani, N. Nagaoka, T. Noda, T. Matsuura, A simple and efficient method for including frequency-dependent effects in transmission line transient analysis, Int. J. Electr. Power Energy Syst. 19 (1997) 255-261.
[11] M.C. Tavares, J. Pissolato, C.M. Portela, New multiphase mode domain transmission line model, Int. J. Electr. Power Energy Syst. 21 (8) (1999) 585-601.

[12] M.S. Sarto, A. Scarlatti, C.L. Holloway, On the use of fitting models for the time-domain analysis on problems with frequency-dependent parameters, in: Proceedings of the IEEE International Symposium on Electromagnetic Compatibility, Montreal, Quebec, Canada; August, 2001.

[13] B. Gustavsen, Validation of frequency dependent transmission line models, IEEE Trans. Power Deliv. 20 (2) (2005) 925-933.

[14] S. Kurokawa, F.N.R. Yamanaka, J.A. Prado, J. Pissolato, Inclusion of the frequency effect in the lumped parameters transmission line model: state space formulation, Electr. Power Syst. Res. 79 (7) (2009) 1155-1163.

[15] P.T. Caballero, E.C.M. Costa, S. Kurokawa, Fitting the frequency-dependent parameters in the Bergeron line model, Electr. Power Syst. Res. 117 (2014) $14-20$.

[16] E.C.M. Costa, S. Kurokawa, A.J.G. Pinto, B. Kordi, J. Pissolato, Simplified computational routine to correct the modal decoupling in transmission lines and power systems modeling, IET Sci. Meas. Technol. 7 (1) (2013) 7-15.

[17] R.C. Silva, E.C.M. Costa, S. Kurokawa, J. Pissolato, Mutual coupling modeling in transmission lines directly in the phase domain, in: Proceedings of the IEEE Electrical Power and Energy Conference-EPEC, Winnipeg, MB, Canada, 2011, pp. 486-491. 